\title{
Téoros
}

Revue de recherche en tourisme

\section{Les centres de référence en tourisme}

\section{Yvan Dubé}

Volume 1, numéro 2, 2e trimestre 1982

La recherche en tourisme : forces et faiblesses

URI : https://id.erudit.org/iderudit/1080856ar

DOI : https://doi.org/10.7202/1080856ar

Aller au sommaire du numéro

Éditeur(s)

Université du Québec à Montréal

ISSN

0712-8657 (imprimé)

1923-2705 (numérique)

Découvrir la revue

Citer ce document

Dubé, Y. (1982). Les centres de référence en tourisme. Téoros, 1(2), 20-23.

https://doi.org/10.7202/1080856ar d'utilisation que vous pouvez consulter en ligne.

https://apropos.erudit.org/fr/usagers/politique-dutilisation/ 


\section{Les centres de référence en tourisme \\ Par Yvan Dubé}

Nous vous présentons ungrand nombre de centres de référence auxquels vous pourrez avoir recours lors de vos recherches en tourisme. Dans l'ensemble, ces différents centres ont pris naissance il y a dix ans ou moins. Certains de ces centres sont très bien équipés matériellement (informatique, cartothèque, microfiches). Ils sont sous la responsabilité de bibliothécaires professionnels ou de documentalistes d'expérience prêts à collaborer avec vous.
A la suite de cette recherche, nous pouvons affirmer, que nous disposons, au Québec, d'une mine de renseignements absolument vitale à l'avancement de la "science touristique" ou de la "téorologie".
L'accessibilité à ces services est quelquefois restrictive. II vous faudra faire une demande verbale a la personne responsable. Vous pourrez avoir à vous déplacer soit à Granby, Sherbrooke, Québec, Chicoutimi. Ces centres sont ouverts du lundi au vendredi et certains sont mêmes ouverts le samedi. Ils sont pourtant, pour la plupart, sous-utilisés.
Centre d'études du tourisme

1420 , rue St-Denis

Montréal $\mathrm{H} 2 \mathrm{X}$ 3J8

282-9613 ou 9614

C.P. 8000 , Succursale A

Montréal H3C $3 \mathrm{~L} 4$

Jean Pelletier

$9 \mathrm{~h}$ a $18 \mathrm{~h}$ (19h les mardis et jeudis)

Membre du Bureau international du tourisme social (B.I.T.S.)

Membre de I'Organisation mondiale du tourisme (O.M.T.)

Membre du Tourisme Travel Research Association (T.T.R.A.)
- 12000 documents: études statistiques, techniques, juridiques, résultats d'enquêtes et de recherches, plans de développement et d'aménagement et, ce, dans la plupart des secteurs d'actitivés touristiques.

- environ 300 périodiques spécialisés
Statistiques Québec

1. Place Ville Marie, $14 \mathrm{e}$ étage

Montréal H3B 2B2

873-4722

Lise Côté

$9 \mathrm{~h}$ à $12 \mathrm{~h}-13 \mathrm{~h} 30$ à $16 \mathrm{~h} 30$
- bilans statistiques sur le loisir, la chasse et la pêche

- statistiques sur l'hỏtellerie

- dossiers techniques sur "les habitudes de vacances des familles"
Statistiques Canada

Plaza Alexis Nihon

1500, Atwater

Montréal $\mathrm{H} 3 \mathrm{Z} 1 \times 5$

283-5725

Paul Baril

9 h à 16 h 30
- dossiers techniques sur des "énquêttes sur les voyages des canadiens". Dossier 66201: voyages entre le Canada et les autres pays; dossier 87401 : voyages, tourisme, loisir de plein-air
Division de la recherche et de l'analyse socio-économique (M.L.C.P.)

150 est, boul. St-Cyrille

7e étage, Québec, G1R 4 Y 1

418-643-2264

Robert Petit Clair

$9 \mathrm{~h}$ à $12 \mathrm{~h}-13 \mathrm{~h} 30$ aे $16 \mathrm{~h} 30$
- système d'inventaire des ressources touristiques et des équipements de loisirs de plein-air (+le socio-culturel) (S.I.R.T.E.L.)

- banque de données, mise à jour annuellement, de plus de 12500 établissements et installations québécoises de tourisme et de loisirs de plein-air et de 100 types d'équipements avec les caractéristiques qui s'y rattachent. 
Centre de références et de donnés touristiques

Office de tourisme du Canada

235 ouest, Queen, $3 e$ étage

Ottawa, Ontario K1A OH6

613-995-2754

Réal Viau

$7 \mathrm{~h} 30$ a $17 \mathrm{~h}$

5000 livres et documents:

- service public qui coordonne et qui emmagasine les differentes données touristiques pour l'ensemble du Canada. II regroupe aussi d'autres sources d'informations tant américaines qu"internationales

- publication chaque année du catalogue de livres des études de recherches de tourisme (ce catalogue est gratuit)

Centre de documentation du Secrétariat des organismes de loisir du Québec

1415 est, rue Jarry

Montréal H2E $2 Z 7$

374-4700

Martine Paquet-Lahait

$9 \mathrm{~h}$ aे $12 \mathrm{~h}-13 \mathrm{~h}$ à $17 \mathrm{~h}$
- domaine du loisir: philosophie, sociologie, documents techniques, équipements en loisir et aussi le plein-air
Centre de recherche sur les transports

Université de Montréal

3535. Queen Mary, bureau 430

Montréal H3V $1 \mathrm{H} 8$

343-7575

Jean-Marc Rousseau

$9 \mathrm{~h}$ à $17 \mathrm{~h}$
- transport ferroviaire

- transport de marchandises

- transport urbain
Centre de sondage

Université de Montréal

5858. Côte des Neiges

Montréal H3C 3J7

343-7596

Victor Tremblay

$9 \mathrm{~h}$ a $17 \mathrm{~h}$
- banque de données

- catalogues

- dossiers techniques sur les parcs dans la région de Montréal
Département de géographie

Bibliothèque et cartothèque

Université de Montréal

520. Côte Ste-Catherine

Salle 339

Montréal H2V 2B8

270-3727

Francine Caplette

Été: 9 h à $12 \mathrm{~h}-13 \mathrm{~h} 30$ à $17 \mathrm{~h}$

Aut: 9 h à $20 \mathrm{~h}$ (lundi au jeudi)

$9 \mathrm{~h}$ à $17 \mathrm{~h}$ (vendredi)

$12 \mathrm{~h}$ a 17 (samedi)
- système informatisé

- géographie touristique (secteur négligé)

- la géographie humaine et la géographie physique sont les principaux sujets de référence

\section{INRS-Urbanisation de I'UQAM}

3465, Durocher

Montréal $\mathrm{H} 2 \times 2 \mathrm{C} 6$

842-4191

Hélène Houde

$9 h$ à $12 \mathrm{~h}-13 \mathrm{~h} 30$ à $17 \mathrm{~h}$
- centre de documentation et de cartographie

- badaduq, service informatisé de la bibliothèque de I'UOAM

- dossiers techniques: "vancances et tourisme 1980 " de Marcel Samson et "les parcs urbains à Montréal" de Marcel Gaudreau
Centre de documentation Parcs-Canada

1141, route de l'Église

Ste-Foy, G1V $4 \mathrm{H} 5$

418-694-7390

Cécile Morin

$8 h 30$ à $12 \mathrm{~h}-13 \mathrm{~h} 30$ à $16 \mathrm{~h} 30$
8000 documents:

- équipement

- environnement

- aménagement

- parcs

- urbanisme

- plein air 
Bibliothèque de I'I.T.H.Q.

401. De Rigaud, 1er étage

Montréal H2T $1 \mathrm{E} 6$

873-4163 poste 126

Céline Beauchemin

$8 \mathrm{~h}$ à $12 \mathrm{~h}-13 \mathrm{~h}$ à $16 \mathrm{~h}$
10000 documents:

- on peut y consulter les travaux du Centre de recherches technologiques

- hơtellerie

- restauration

- hospitalité

- gestion hôtelière

- marketing et publicité
Office de planification et de développement du Québec 440 ouest. Dorchester

Montréal H2Z $1 \mathrm{V7}$

873-5845

Alice Lemire

$8 h 30$ à $12 h-13 h 30$ a 16 h 30
3500 documents:

- développement régional

- aménagement du territoire

- municipalités régionales de comtés (M.R.C.)
Organisation de l'aviation civile internationale 1000 ouest. Sherbrooke

Montréal H2L 1 L5

285-8219

Fathia Ismail

$8 h 30$ a $16 h 30$
7000 volumes (surtout en anglais):

- transports internationaux

- législation internationale
Bibliothèque. Cegep de Granby

235. St-Jacques

Granby, J2G 3N1

514-372-6614

Réjean Normandin

$8 \mathrm{~h}$ à $12 \mathrm{~h}-13 \mathrm{~h}$ a $17 \mathrm{~h}$
- travaux d'étudiants en techniques de tourisme, depuis 1970

- audiothèque avec 20,000 diapositives et vidéo

\section{Air Canada}

Services auxiliaires

Place Ville Marie

38 e étage, Montréal

H3B 3P7

874-4842

Iris Land

$8 \mathrm{~h} 30$ à $16 \mathrm{~h} 30$
25000 volumes:

- transports (économie du transport, management)

- 6 personnes à plein temps

- système informatisé

\section{Téléglobe Canada}

Services auxiliaires

Montréal H3A 2 S4

281-5210

Hêlène Dumont

$8 \mathrm{~h} 30$ 青 $16 \mathrm{~h} 30$
680 ouest, Sherbrooke

10000 volumes:

- télécommunications

- finances

- marketing

- informatique

32000 documents:

Bibliothèque Dechief

Service de la gestion

Canadien National

935 ouest, de la Gauchetière

Montréal H3B 2M9

877-5234

Katlin Elliot

$8 \mathrm{~h} 30$ a 17 h
- transport de passagers

- transport de marchandises

- télécommunications

- chemins de fer (I'histoire des) 
Centre de documentation

Hydro-Québec

75 ouest. Dorchester

Montréal H2Z $1 \mathrm{~A} 3$

289-2316

Jocelyne Bisaillon

$8 h 30$ aे $16 h 30$
- énergie

- cartes

- services publics

N.B.: présentement ce service est en réorganisation administrative

\section{Bibliotheque centrale}

Services administratifs

Canadien Pacifique

Gare Windsor, rue de la Gauchetière ouest

H3C 3E4

295-6762

Anita Di lorio

9h à $17 \mathrm{~h}$
10500 documents, 450 périodiques:

- système informatisé

- transport, énergie, finance

- consultation sur demande spéciale

\section{Centre d'information spécialisée}

Bell Canada

1050. Cóte du Beaver Hall

Montréal H2Z $1 \mathrm{~S} 3$

870-8500 ou 3364

Rhonda Greenfeld

$8 \mathrm{~h} 30$ à $17 \mathrm{~h}$
- consultation sur demande spéciale

- le système de recherches est informatisé

- services publics

- télécommunications

\section{Daniel Arbour et André Marsan}

1210 ouest. Sherbrooke

Montréal H3A $1 \mathrm{H} 7$

288-1294

Louis Rousseau

Bh a 16 h 30
6000 volumes:

- urbanisme

- environnement

- loisir

- géographie

(associés de Lavalin)

\section{Éconosult (Lavalin)}

1210 ouest, Sherbrooke

Montréal H3A $1 \mathrm{H} 7$

288-4917 poste 2859

Carole Urbain

gh à $17 \mathrm{~h}$
3500 volumes:

- economie du tourisme

- développement régional

- consultation sur demande spéciale
Suite... en bref

Dans l'énumération précédente des différents centres de référence ayant un rapport direct ou indirect avec le tourisme, nous n'avons pu faire le tour de tous les systèmes de référence disponibles. De là l'idée d'inscrire, à cette liste, d'autres centres où vous pourrez trouver une information pertinente et de bonne aloi.

Cinémathéques de la ville de Montréal, du Québec, de l'Office national du film.
Service d'Archives de la ville de Montréal, Archives nationales du Québec.

Bibliothèque de la ville de Montréal, Bibliothèque nationale (St-Sulpice).

Centre de recherche en aménagement régional, département de géographie, Université de Sherbrooke.

Groupe d'étude et d'intervention régionales. Université du Québec à Chicoutimi (UQAC). Jean Désy. Décembre 1980.
Centre de recherche en développement économique (C.R.D.E.). Université de Montréal.

Centre de recherche en aménagement et en développement (C.R.A.D.) Université Laval.

Certaines agences de publicité (B.C.P., Cossette, Communicateurs PLT, etc.).

Bibliothèque de I'Université McGill (programme de 2e cycle en tourisme).

Centre d'études en loisir. Université du Québec à Trois-Rivières (UQTR) 